\title{
Dust-formation episode of the long-period WC+O binary WR 137: direct imaging with HST-NICMOS2
}

\author{
Sergey V. Marchenko, Anthony F.J. Moffat, and Yves Grosdidier \\ Département de Physique, Université de Montréal, Québec, Canada
}

\begin{abstract}
We have resolved, for the first time in any WR+OB system, IRemitting dust in the close environment of the long-period binary WR 137 . The dust emission occurs in a few clumps within about 0.5 of the star, as well as in a jet-like structure with total extension of about 0 !' 25 .
\end{abstract}

\section{Observations}

Recent observations (Williams 1996 and these Proceedings) showed that the IR flux of one of the seven dust-forming WR+O systems ( $c f$. Williams 1995), WR 137 (HD 192641, WC7+OB) was on the rise again, a dozen years after a spectacular culmination of dust formation in 1984.4 (Williams et al. 1985). We have used HST-NICMOS2 (0.'075/pixel scale) with the medium-band filters F165M $\left(H^{\prime}, \lambda_{c}=1.65 \mu \mathrm{m}\right)$ and F237M $\left(K^{\prime}, \lambda_{c}=2.37 \mu \mathrm{m}\right)$ to observe WR 137 and WR 138 (the latter as a point-spread-function reference star) for two orbits on 10 September 1997 (around the maximum of the IR $K$-band flux: Williams 1998, private communication), and for one orbit on 18 May 1998.

\section{Results}

The estimated $H^{\prime}$ magnitudes (6.74 in 1997 and $6.76 \pm 0.03$ in 1998) closely match the values obtained during the previous 1984.4 maximum (Williams $\mathrm{et} \mathrm{al}$. 1985 ), while the $K^{\prime}$ values (5.94 in 1997 and $6.06 \pm 0.03$ in 1998) slightly exceed the corresponding broad-band $K$ fluxes, as expected for a system containing a copious amount of heated dust with $T_{\mathrm{d}} \leq 10^{3} \mathrm{~K}$.

After maximum entropy (ME) image restoration (Cornwell \& Evans 1985), we can see three distinct clumps in the $1997 K^{\prime}$ image of WR 137: summed over all three clumps, $K^{\prime}(1997)=10.7 \pm 0.4 \mathrm{mag}$. Two of the clumps disappeared about eight months later, while the persistent clump $\left(K^{\prime}=13.1\right.$ mag $)$ receeded away from the star (Fig. 1) at $i=68^{\circ} \pm 10^{\circ}$ relative to the plane of the sky. The central source on the $1997 H^{\prime}$ and, especially, $K^{\prime}$ ME-images, is elongated, with the major axis pointing to the ejected clumps. The $1998 K^{\prime}$ ME-image shows a spectacular, bright jet with integrated flux of $K^{\prime}(1998)=7.6 \pm 0.3 \mathrm{mag}$, moving at $i \approx 27^{\circ}$ relative to the plane of the sky. Clearly seen in the $1998 H^{\prime}$ image is a hot clump ( $\left.T_{\text {clump }} \geq 2200 \mathrm{~K}\right)$ positioned right at the jet's extremity.

Assuming the dust to be amorphous carbon (Williams et al. 1987; Zubko 1998 ) and adopting $T_{*}=35000 \mathrm{~K}, R_{*}=10 \mathrm{R}_{\odot}$, and $\mathrm{d}=1.82 \mathrm{kpc}$ (van der Hucht et al. 1988), we find: (a) $M_{\mathrm{d}} \simeq 5.410^{-9} \mathrm{M}_{\odot}$ for the two non-persistive clouds in 


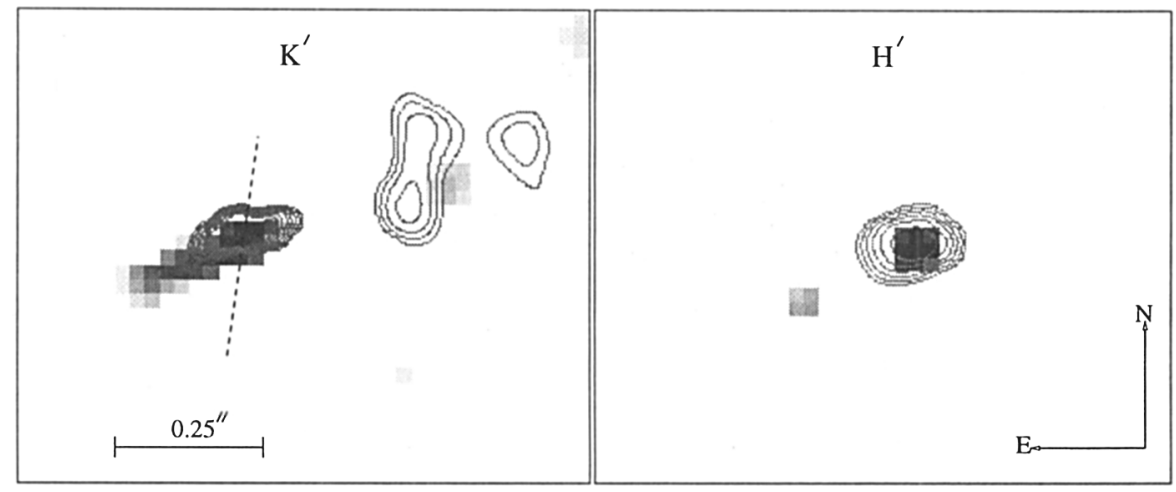

Figure 1. The HST-NICMOS2 ME-restored, factor-of-3 sub-sampled images of WR 137: gray-scales show the $1998 H^{\prime}$ and $K^{\prime}$ log-scaled observations; the contours depict the $1997 H^{\prime}$ and $K^{\prime}$ images. The dashed line indicates the probable orientation of the projected axis of symmetry of the flattened WR wind, based on spectro-polarimetry of the WR star.

1997; (b) $M_{\mathrm{d}} \simeq 2.310^{-7} \mathrm{M}_{\odot}(1997) \simeq 2.210^{-7} \mathrm{M}_{\odot}$ (1998) for the persistent cloud; and (c) $M_{\mathrm{d}} \simeq 1.710^{-9} \mathrm{M}_{\odot}$ for the jet; all the masses are uncertain by a factor 3 .

The dust formation and its spatial distribution in WR 137 are governed by four main factors: (1) by the changing orbital separation of the components around periastron; (2) by instabilities in the WR wind and/or instabilities in the wind-wind collision zone; (3) by instability-related shocks leading to strong temperature fluctuations; and (4) by an additional density enhancement from the flattened WR wind (Harries et al. 1998). All four factors combined together could lead to a $\frac{\rho}{\rho_{0}} \simeq 10^{3}$ gain in the gas density for clumps formed from gas compressed by the wind-wind collision, thus substantially facilitating the creation of dust.

\section{References}

Cornwell, T.J., Evans, K.F. 1985, A\&A 143, 77

Harries, T.J., Hillier D.J., Howarth, I.D. 1998, MNRAS 296, 1072

van der Hucht, K.A., Hidayat, B., Admiranto, A.G., Supelli, K.R., Doom, C. 1988, A\&A 199, 217

Williams, P.M. 1995, in: K.A. van der Hucht \& P.M. Williams (eds.), Wolf-Rayet Stars: Binaries, Colliding Winds, Evolution, Proc. IAU Symp. No. 163 (Dordrecht: Kluwer), p. 335

Williams, P.M. 1996, in: J.-M. Vreux, A. Detal, D. Fraipont-Caro, E. Gosset \& G. Rauw (eds.), Wolf-Rayet Stars in the Framework of Stellar Evolution, Proc. $33^{\text {rd }}$ Liège Int. Astroph. Coll. (Liège: Univ. de Liège), p. 135

Williams, P.M., Longmore, A.J., van der Hucht, K.A., Talevera, A., Wamsteker, W.M., Abbott, D.C., Telesco, C.M. 1985, MNRAS 215, 23P

Williams, P.M., van der Hucht, K.A., Thé, P.S. 1987, A\&A 182, 91

Zubko, V.G. 1998, MNRAS 295, 109 